\title{
Information Management for Intelligent Retail Environment: The Shelf Detector System
}

\section{Emanuele Frontoni ${ }^{1, *}$, Adriano Mancini ${ }^{1}$, Primo Zingaretti ${ }^{1}$ and Valerio Placidi ${ }^{2}$}

1 Dipartimento di Ingegneria dell'Informazione (DII), Università Politecnica delle Marche, Via Brecce Bianche, 60131 Ancona, Italy; E-Mails: mancini@dii.univpm.it (A.M.); zinga@dii.univpm.it (P.Z.)

2 Grottini Lab srl, Via S.Maria in Potenza, 62017, Porto Recanati (MC), Italy;

E-Mail: valerio.placidi@grottinilab.com

* Author to whom correspondence should be addressed; E-Mail: frontoni@dii.univpm.it; Tel.: +39-071-2204458; Fax: +39-071-2204224.

Received: 5 December 2013; in revised form: 16 April 2014 / Accepted: 21 April 2014 / Published: 5 May 2014

\begin{abstract}
Shelf-out-of-stock is one of the leading motivations of technology innovation in the shelf of the future. The Shelf Detector project described in this paper aims to solve the problem of data knowledge in the shelf-out-of-stock problem. This paper is mainly focused on the information layer of the system and main novelties illustrated in this work are in the information field demonstrating the huge number of insights that can be derived from the use of such a tool able to gather data in real time from the store. The tool presented is the first being installed for a long time in a high number of stores and products, demonstrating the ability to gather data and extract interesting insights. This paper aims to demonstrate the feasibility and the scalability of our system in providing a high number of data and interesting insights for store and marketing teams. The cloud based architecture developed and tested in this project is a key feature of our system together with the ability to collect data from a distributed sensor network.
\end{abstract}

Keywords: out-of-shelf; automatic detection; distributed sensors networks; shelf-detector system 


\section{Introduction}

Out-of-stock (OOS) events occur when a product is unavailable on shelf for customer purchase. This incident has potentially negative implications for retailers and manufacturers. In fact, when a shopper is faced with an OOS situation, he chooses an alternative action since he cannot purchase the product he desires. In an OOS situation, the consumer generally decides to either purchase a product of another brand, change stores, delay the purchase, or cancel the purchase [1]. These choices are strictly related to the product loyalty and/or the brand loyalty causing loss of sales for both the retailer and the manufacturer. For this phenomenon, an industry body, Efficient Consumer Response (ECR) establishes the industry has a loss of Euro 4 billion each year [2]. Therefore, the risk of the manufacturers is greater than the retailers since the majority of customers are willing to substitute a different brand or stocking keeping unit (SKU) when faced with an OOS situation. Certainly, it is important for brands that their product is available on the shelf whenever the store is open, since it is very difficult to know when the product is purchased.

For about 50 years, a lot of works in academia and industry have dealt with the OOS problem $[3,4]$. OOS has been studied considering three different approaches: the extent [2,5], the consumer responses [1,6] and the logistical issue [7]. In this work, we focus the attention on OOS extent that measures average OOS levels.

The traditional definition of OOS had a limitation since it did not consider the measurement of time related variables. The OOS has been defined as percentage of SKU in a category not available on shelf for consumer purchase [2,5]. Recently, the wireless technology has allowed overcoming time limitations, letting us know the exact moment the product is OOS, its duration and the exact moment when the product was replenished on shelf. Therefore, the introduction of time variable introduces a new definition of OOS related to time: OOS is the percentage of store opening time per day in which consumers would encounter an OOS in a category. Because establishing OOS events in moments of the day, days of the week and the number of facings is a very important aspect for the industry, this paper aims to explore OOS for day of the week, time of the day and number of facings.

The variable OOS by day of the week was analyzed in some studies [2,5,8]. According Andersen and Gruen et al., Monday and Sunday had high value of OOS; in particular, Monday had the highest OOS level. Instead, according to ECR Europe, the highest value of OOS occurs on Friday, followed by Saturday and Tuesday. These differences depend on the retail stores opening in a different country. For all studies, the days with the greatest value of OOS are near weekends. Moreover, some studies $[5,8]$ have examined OOS by time of day, so the variation of OOS during the day. They all suggest that the peak of OOS occurs in the afternoon; the peak increases as day progresses. The number of product facing refers to the number of items, per SKU, seen on shelf from a perspective of consumers [9]. The space available on the shelf represents a fundamental aspect in the distribution of the product, so the number of facings must be considered because it directly affects the likelihood that a product will become OOS. This happens since by increasing the number of facings, the probability of an OOS occurring decreases because more items must be sold. The number of facings influences the purchase of a particular brand and the product having a high impact on consumer attention. Typically, big brands have a larger number of facings than small brands, because the big brands replenish retail stores more frequently, reducing OOS events. 


\section{OOS Extent}

As said, previous studies had not considered time variables to conceptualize OOS events. This was strictly related to measurement systems not able to produce continuously real-time measurement of OOS. Looking at the methods that identify OOS events, note that they have limitations by providing only a snapshot of OOS events and introducing human inaccuracy. In the present research, we introduce a measurement system that takes into account time variable, knowing in real-time OOS events and then promptly operate to solve the problem. The new system allows calculating the OOS taking into account the time variable, by applying a new metric for the OOS detection systems.

Physical audits have been widely used both in industry [5,8] and academia [7], as benchmarks to evaluate the availability of products on shelves. During the physical audit, a group of auditors execute programmed visits in the store and registers which products are not on shelves. During the physical audit, a group of auditors executes pre-planned visits in the store and registers that products are not on shelves. Just a sample of products is chosen for audit, this choice depends on the representativeness of products and the policy of store: all this affects the results and their generalization. This method has some limitations since the observations are recorded only at a specific moment of a particular day, providing a snapshot of the OOS events and not a real-time measurement [5]. The number of audits and the frequency at which they occur vary significantly between studies, thus impacting on the precision of the measure. Clearly, increasing number and frequency, the OOS measure becomes more careful and more representative of the real situation; however drastically increasing the costs. Although the increased frequency of audits determines a full picture of the OOS situation, it is not able to provide a continuous measure of OOS, since it is not known whether a product is still in stock, has been replenished and/or has become OOS.

Another OOS measure is to obtain information from customers, interviewing them directly about their experiences of availability of products. Generally, interviews and surveys occur on the store asking customers if they found all the products that they desired to purchase and so if they were available on shelf. This approach lacks objectivity, since the consumer refers to his favourite item, not providing information about other items in the category. So, it depends on the perception of consumer and his availability. Consumers will have to report to the interviewer their approach to the purchase, remembering a-posteriori their behaviour. Nenycz-Thiel et al. have demonstrated the inaccuracy of data obtained during the interviews and the surveys [10]. The values of OOS produced from research that use consumer interviews or surveys are the percentage of consumers who declared that their favoured product is not on shelf, rather than the percentage of SKUs not available on shelf at the time of purchase.

Some techniques for consumer interviews include also asking store staff and managers questions about OOS levels. Since these surveys can be effective at measuring staff attitudes to OOS situations, they are flawed in that they rely on human knowledge and recall. For instance, a survey asking staff to estimate OOS levels assumes that team members know enough about inventory and ordering processes and that they remember this information and can synthesize it into a single OOS measure.

This paper wants to overcome limitations described in the previous methods by considering a continuous and in real-time measure of OOS events. In fact, the system proposed is automatic and wireless reducing the limitations deriving from physical audits, consumer and staff interviews. So, the 
system can measure the exact moment the product is no longer available on shelf, the exact moment the product is back on shelf and the duration in minutes of OOS. The main goal of this measure is to give back to the retailer or producers an exact measure of shelf-out-of-stock to consider, with real data coming from a real store, how many hours products are not available for shoppers.

The system described is the automatic Shelf Detector system designed in cooperation between Università Politecnica delle Marche and Grottini Lab that overcomes all the discussed issues providing real data in real time, automatically grabbing data from shelves. The system is installed and functioning in six countries, in 50 stores and detects OOS of 4000 products performing a continuous monitoring. Preliminary studies in intelligent retail environments allowed to test the system [12-14], considering also other aspects and applications of this approach in different situations.

\section{The Shelf Detector System}

The major barrier to improvement has been the lack of robust, inarguable measures that could provide indisputable evidence and actionable data at a low cost. Shelf Detector (SD) goes on that direction and provides real data on real time using a small battery based sensors on a Wireless Sensors Network to measure on shelf availability in real time. The system provides also insights on all causes described before, so the next section will provide detail on that.

\subsection{Shelf Detector Hardware}

The system is described as a wireless sensor network (WSN) consisted of spatially distributed autonomous sensors. The star topology of WSN allows to achieve a high degree of scalability and a high level of controllability, as already verified in previous studies [15,16]. Inside a point of sale, several different RF boards can be installed interconnected to the wireless control gear via the $433 / 868 / 915$ radio frequency communication that is actually unused by the other monitoring systems already in stores. Each RF board can manage up to 200 sensors. Basically, there is a sensor per facing. Every SKU is typically linked at one EAN code (unique identifier) of the respective facing package. The sensor technology is based on the transmission and the detection of the IR signal when the target reflects it; so, every item is sensed by the detection of IR signal reflection transmitted by the sensor. The percentage of IR signal reflected by the target is then detected by the sensor to calculate the numerical level of proximity. If the level proximity goes under a threshold value, the item is marked as absent. The sensors monitor physical or environmental conditions, such as temperature, sound, pressure, etc. and pass cooperatively data through the system to the main location.

Figure 1 shows in detail the components of the shelf detector system. Moreover, technical specifications are as follows:

(A) Sensors-IR detection, 5V DC, dimensions 1" × 0.4 "

Bars-linear modular profile, dimensions (depth) $0.6^{\prime \prime} \times$ (height) $1.6^{\prime \prime}$

(B) RF-Radio Frequency Transmitter, 433/868/915 MhZ, 2 long life torch batteries (D-R35), dimensions $4.5^{\prime \prime} \times 4.5^{\prime \prime} \times 2.2^{\prime \prime}$

(C) Control Gear-85/264V AC-45/440 Hz power, GSM/Gprs DualBand modem, RadioFrequency receiver, 433/ 868/915 MhZ, dimensions 5.2" × 4" × 1.2". 
Figure 1. List of the components of the Shelf Detector (SD) system.

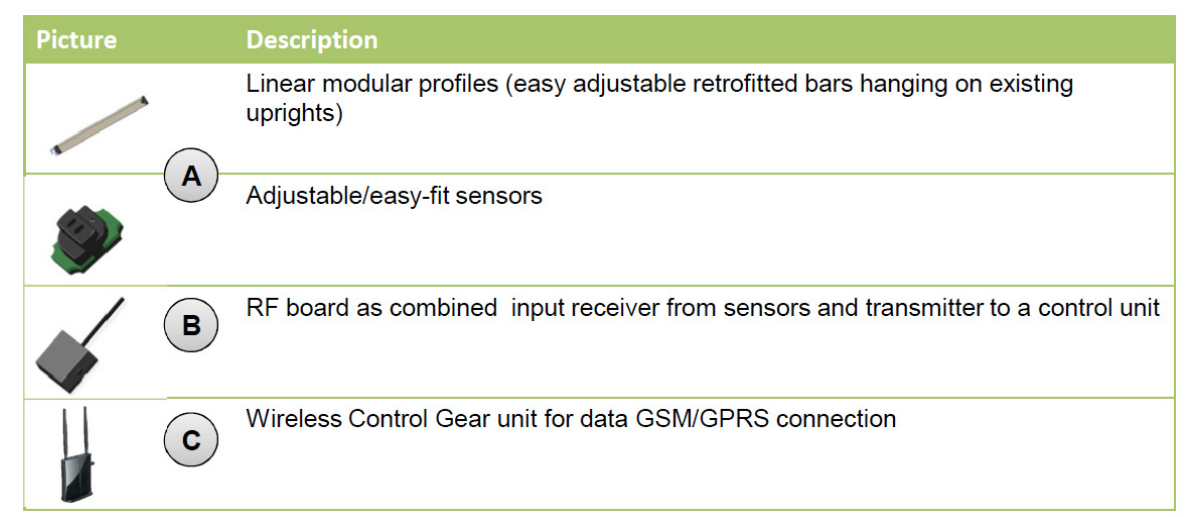

The installation on the shelf is based on a generic hanging system that can connect bars on every shelf. The installation is very easy and fast in each store. The following Figure 2 shows the installed system. The sensors can be easily installed and can be directly removed through the bar. The system is totally based on battery power apart from the Control Gear (one per store able to transmit data on GPRS/UMTS). "Battery's life" means the time your system will run before it is recharged. The system is powered by two standard torch batteries: alkaline battery that provides 18 Amp hours. The duration of the two batteries inside the RF box is enough to power up to 200 sensors for a year.

Figure 2. Particular of the generic installed hanging system.

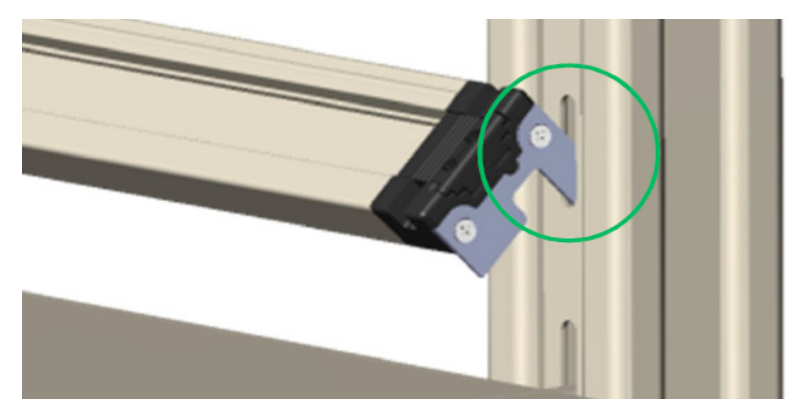

The system is really easy to install and to maintain. The installation time can be better explained using an example: the installation of a category with about 500 sensors (SKUs), removing products from the shelf, installing bars and sensors, testing the system and defining the planogram on the software take about four hours. No maintenance was required for an installation that has been online from more than two years. The system is patented worldwide, designed and maintained from Italy, produced in the Far East. After one year, the shelf detector system was installed in different retail stores in the USA, Spain, France, Switzerland and Italy. This pilot phase intended to evaluate the feasibility of monitoring item availability at retail stores obtaining the inventory level of examined products. The panel of products to be monitored during the pilot study was jointly defined with the board of companies to be representative of the items manufactured by those companies.

\subsection{Shelf Detector Software}

Shelf Detector software is the heart of the shelves management system and is totally web based, accessible from anywhere and hosted on a cloud. 
It provides a comprehensive set of statistics that will be described below. The system is also tablet compliant (Android, RIM and iOS) allowing the use of the system directly in front of the shelf.

The software provides to the users, according to their level, an amount of different features shortly described below and not only directly joined with the OOS problem, but also devoted to store management.

Admin users can:

- access cumulative indicators of the macro performance of stores with OOS;

- know the total amount of OOS hours and the performance of every PoS, every shelf and every category;

- define store names and features (opening time, number and type of shelves, etc.);

- manage a list of mail and mobile numbers for OOS real time notifications;

- edit a product database by brand and product category;

- manage product dimensions for restocking purposes (even with a box restocking logic);

- define a planogram with evidence of monitored products and upgrade it any time;

- edit any OOS to correct or fix them in case of false refilling;

- download raw data about OOS and cumulated data;

- check facings never consumed (to start planning an improved planogram).

Store managers or other users can:

- access cumulative indicators of the macro performance of their store;

- know the total amount of OOS hours in the store and the performance of every shelf and product category;

- access the complete list of OOS and filter by time and category;

- access easy to read weekly statistics to adapt refill time in the store and in the back store;

- produce Out-Of-Stock Checklist available for single store;

- access full information about OOS (time, number of facing, refill time, etc.);

- access the list of out of facings for multi facings products;

- manage planogram changing every facing and every product to adapt the planogram;

- edit a product database by brand and product category.

Cloud computing is the use of computing resources (hardware and software) that are delivered as a service (typically the Internet). End users access cloud-based applications through a web browser or a lightweight desktop or mobile application, while the business software and user's data are stored on servers at a remote location. Cloud computing relies on sharing of resources to achieve coherence and economies of scale similar to a utility (like the electricity grid) over a network. The cloud-based implementation is the better way to store data and analyse them. Also, the web based solution is a quicker way to obtain statistic reports and share them.

\section{Temporal Metrics and Statistics}

The shelf detector system provides useful data to identify continuously in real time OOS events. According to these data, we introduced new measures that take into account time variables. The metrics provided by the European OOS Index [11] differ from our metrics in the following, since they did not take into account time variables. New parameters extracted from data obtained in real time are: 
(1) Shelf-out-of-stock rate [\%] is the number of hours shelf/facings empty for a SKU, divided by the total number of hours of store opening (expressed as a percentage):

$$
\operatorname{SOOS}_{S K U}=\frac{N_{h S K U_{i}}}{N_{h O S}} \times 100
$$

$\mathrm{i}$ index indicates a specific SKU.

(2) Shelf-out-of-stock by SKU [\%] is the number of hours shelf/facings empty for a SKU divided by the total number of hours of shelf-out-of-stock for all SKUs (expressed as a percentage):

$$
\operatorname{SOOS}_{S K U_{i}}=\frac{N_{h S K U}}{\sum_{i=1}^{n} N_{h S K U_{i}}} \times 100
$$

$\mathrm{n}$ index is the total number of SKU.

(3) Shelf-out-of-stock by week day is number of shelf-out-of-stock by week day:

$$
\operatorname{SOOS}_{W D}=\sum_{i=1}^{n} N_{S K U_{i}}
$$

(4) Shelf-out-of-stock by hours is the number of shelf-out-of-stock by hours:

$$
\operatorname{SOOS}_{h}=\sum_{i=1}^{n} N_{h S K U_{i}}
$$

(5) Refilling performance is refill performance by days and hours;

(6) Product availability is a comparison between product availability by day;

(7) Number of hours from empty to full is number of hours of empty facings:

$$
N_{h_{E F}}=N_{h_{F}}-N_{h_{E}}
$$

(8) Average number of hours from empty to full for all incidents:

$$
M N_{h_{E F}}=\frac{1}{A} \sum_{i=1}^{A} N_{h_{E F i}}
$$

where index $\mathrm{A}$ is the number of accidents.

(9) Full Stock Index is the number of hours shelf completely full divided by total store opening time as rate of full stock (all SKUs).

$$
H_{F S}=\frac{N_{h_{F}}}{N_{h O S}} \times 100
$$

\subsection{General Statistics}

Every report can be configured selecting (Figure 3) date range, shelf (representing the category) and several analysis metrics (such as for example: full stock index, SKU performance, SKU availability, etc.). 
Figure 3. Example of report with a product of interest.

\begin{tabular}{|c|c|c|c|c|c|c|}
\hline Date range & 10 June, 2012 - 13 June, 2012 & \multirow{2}{*}{$\begin{array}{l}\text { Compare to } \\
\text { Compare to }\end{array}$} & \multicolumn{3}{|c|}{1 July, $2012-7$ July, 2012} & \\
\hline Shelf & Baby Care (valid from $30 / 05 / 2012$ to $22 / 06 / 2012$ ) & & \multicolumn{4}{|c|}{ Baby Care New WoT (valid from $25 / 06 / 2012$ to ) $\quad *$} \\
\hline \multicolumn{7}{|l|}{ Analysis metrics: } \\
\hline Full Stock Index 0 & $\checkmark$ Average Soos Lenght 6 & \multicolumn{2}{|c|}{$\checkmark$ SKU performance ${ }^{\circ}$} & & & $\checkmark$ \\
\hline SKUs availability ${ }^{\circ}$ & $\checkmark$ Average SKUs avallability ${ }^{\circ}$ & \multicolumn{2}{|c|}{$\checkmark$ Assessment of facing utilisation 0} & & hin product & $\checkmark$ \\
\hline
\end{tabular}

On the basis of the generated report, the following Figure 4 shows the first part of the report and presents some data about the selected product (retailer, store, category and period) and a significant amount of interesting data.

Figure 4. Particular of the report produced.

\begin{tabular}{|ll|}
\hline RETAILER & TEST \\
POS (TYPE) & Store 1 \\
CATEGORY & Baby Care (9 SKU 18 FACING) \\
PERIOD & FROM 06/02/2012 TO 06/15/2012 \\
AVG SOOS LENGHT & $\mathbf{4 7}$ hours $\mathbf{7}$ min \\
FULL STOCK INDEX & $\mathbf{3 5 , 4} \%$ \\
\hline
\end{tabular}

In order to demonstrate the functionality of the SD system, some values of metrics are represented in the figure, as described earlier, referring to a particular product. These (and other) values can be used to evaluate the efficiency of a store in dealing with the out-of-shelf problem.

\subsection{SKUs Performances}

All the graphs presented in this section refer to the performances of the product. They first consider those values of the report from the point of view of the product.

Figure 5. Shelf-out-of-stock rate referring to different SKU.

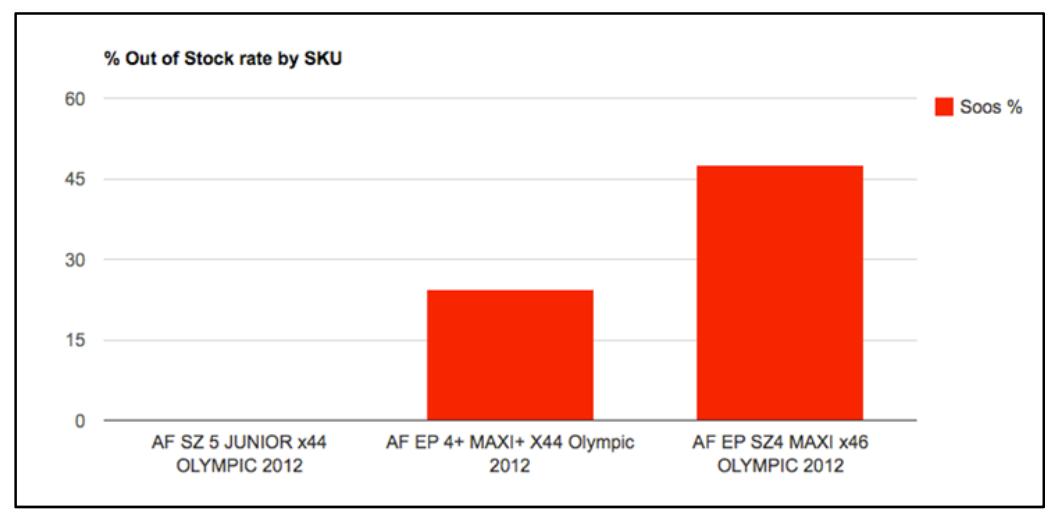


The histogram in figure 5 represents the percentage of out-of-stock considering three different categories of product.

Figure 6. Shelf-out-of-stock by SKU.

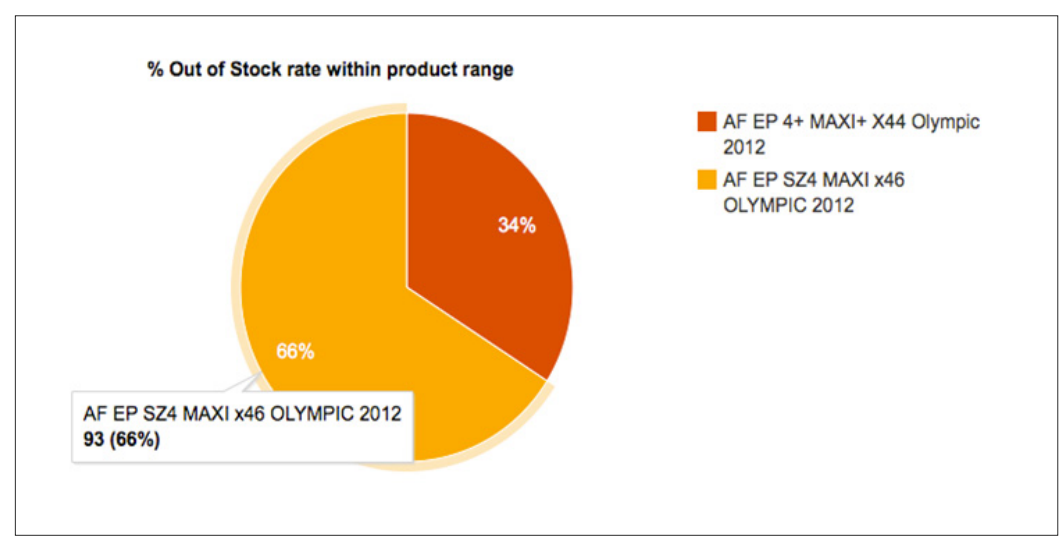

Figure 6 considers the out-of-shelf within a range of products. From Equation (2), we obtain the results for each SKU which are represented in Figure 6. A time-based graph with SKU performances at various time points can be accessed clicking on the bars or on the pie. A pop-up will display a graph as per Figure 7, examining the problem and looking to the OOS periods for each SKU.

Figure 7. The broken line in the graph shows the OOS period for each SKU.

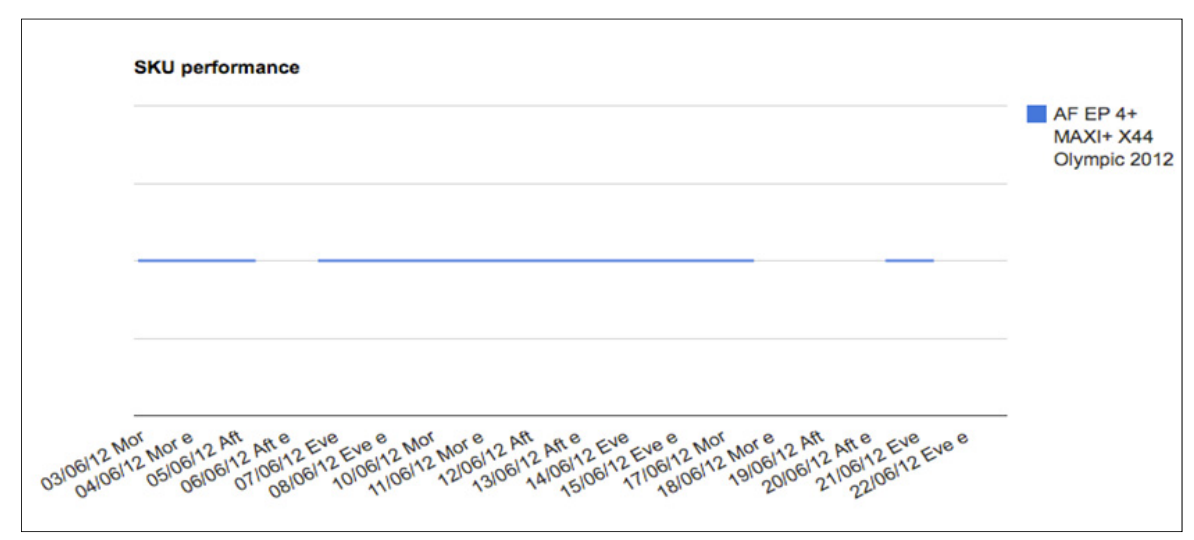

When the product is not out-of-shelf there is the line, while when the product is out-of-shelf there is not.

\subsection{Product Availability}

Figure 8 represents a graph that provides an insight to day by day availability over quite a long period (week or month). The graph, as always, is interactive and you can move the mouse on the line to read values. Thanks to this graph, it is possible to know the amount of product available daily and therefore understand why there may be a drop in product levels on special days. Furthermore, it also provides insights as to when replenishment of a product on the shelf occurs. 
Figure 8. Daily availability of a product.

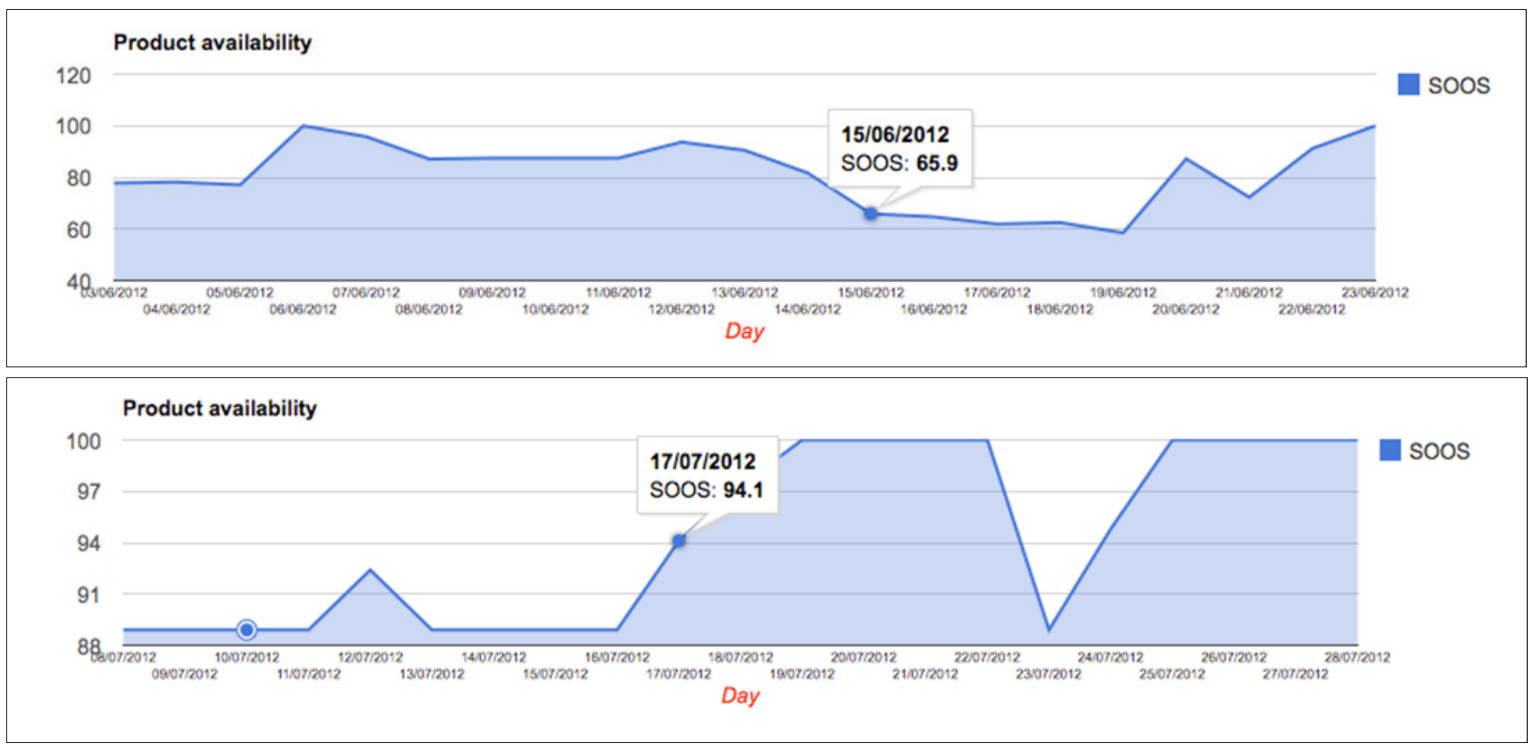

Figure 9 is a lines-based graph where each line is a SKU (defined by a colour joined with a name) that gives an idea of the detail of the SOOS by measuring every SOOS event and refill activity, respectively, and start and end points of each segment. Every day is divided by Morning (Mor), Afternoon (Aft) and Evening (Eve).

This report gives detailed information about a period with respect to the most relevant SOOS accumulated in some days (in the example, the worst period is between the evening of 15 th and the afternoon of the 19th).

Figure 9. The broken line in the graph shows the OOS period for different SKU within the product range.

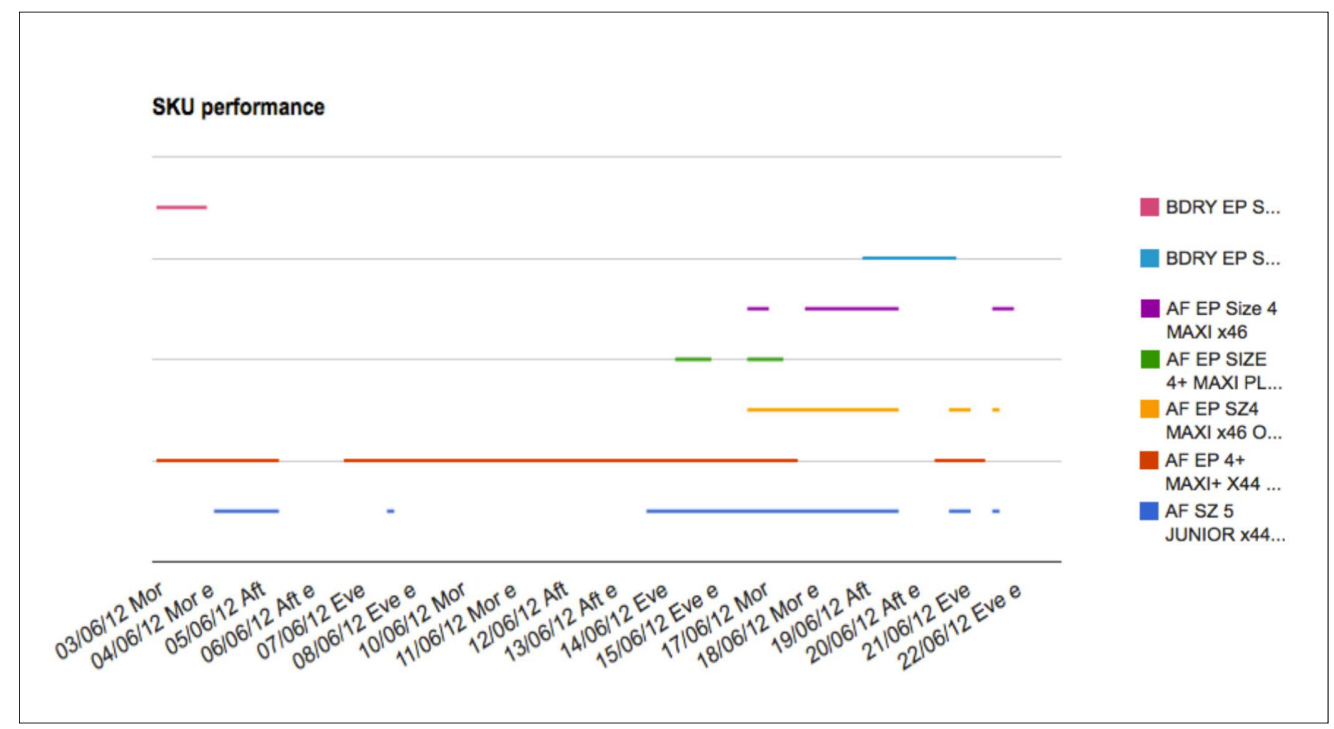

\subsection{Store Team and Shoppers' Behaviors}

Consumers need to find the product they are looking for in store when they need it. If this does not happen, they must then decide what to do - they can buy another brand or try to find the product in 
another store. In order to monitor and then to avoid this kind of situation, the system produces a report to show exactly when the events happen on a daily and hourly basis. This should be useful in creating different refill strategies based on the specific circumstances of the point of sale or based on the specific behaviour of the shopper of a particular store in a specific time of a specific day, thereby finding tailor made solutions to fix SOOS. Figure 10 shows the day of the week and Figure 11 the hour of the day (for a specific store) when the events happen based on the number of SOOS events.

Figure 10. Rate of SOOS related to a week.

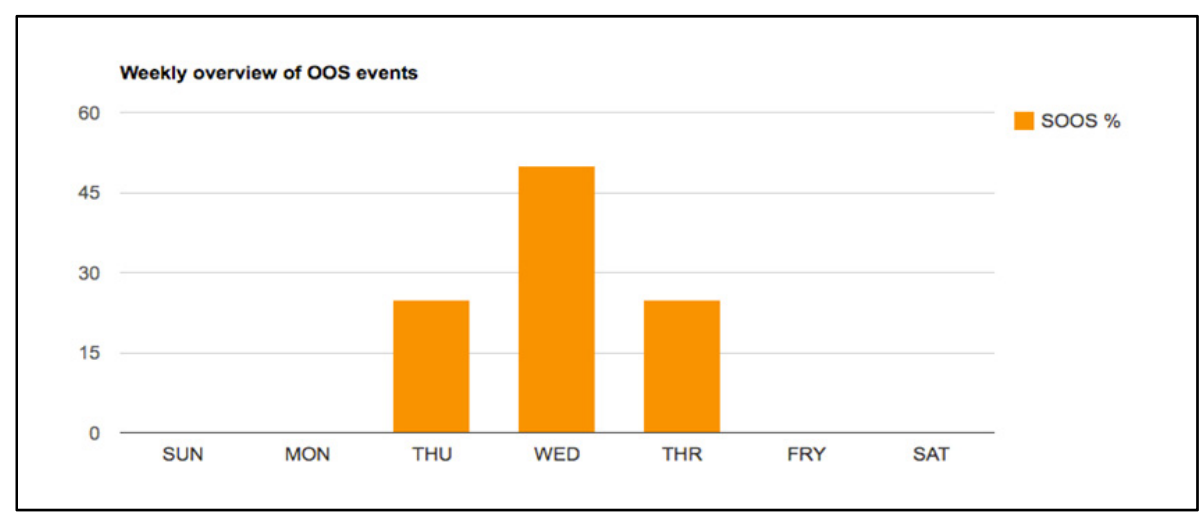

Figure 11. Rate of SOOS related to a day.

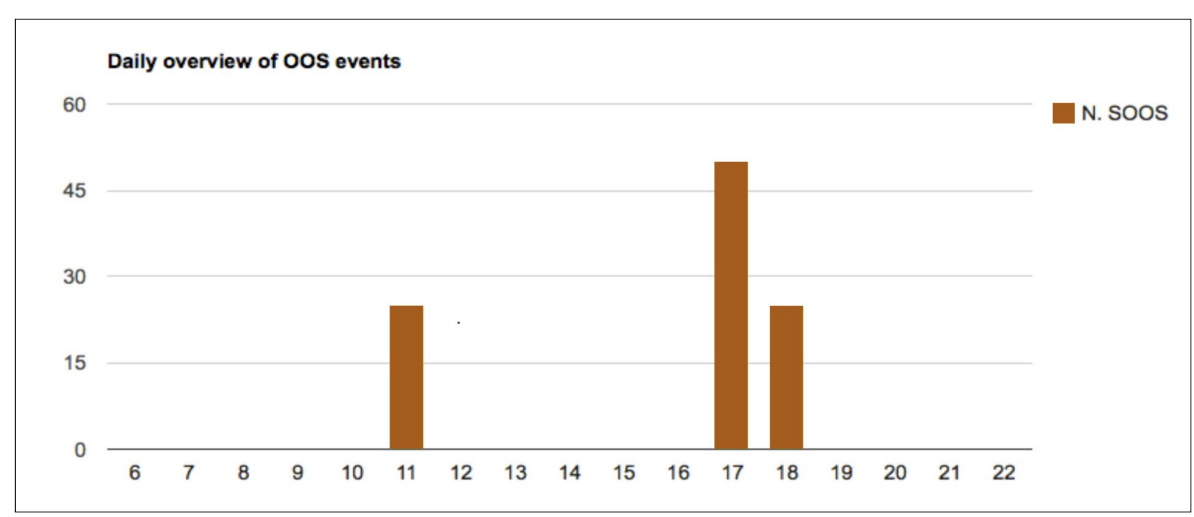

All these reports should be tailor made based on a time period or specific store.

\subsection{OOS Motivations and Promo Periods}

As shown below in Figure 12, the system offers a user interface to select a motivation, in a preordered range, to define the reason why the SOOS occurred. The reason could be that the product is part of a promotional event and/or a bad reordering occurred. There may be no obvious cause, and so on. 
Figure 12. User interface to select the SOOS motivations.

\begin{tabular}{|c|c|c|c|c|c|}
\hline From & To & Product & Shelf & Promo & Motivations \\
\hline $\begin{array}{l}07 / 06 / 2012 \\
17: 15: 58\end{array}$ & $\begin{array}{l}12 / 06 / 2012 \\
10: 42: 19\end{array}$ & AF EP 4+ MAXI+ X44 Olympic 2012 & Baby Care & YES : & Bad Reordering : \\
\hline $\begin{array}{l}12 / 06 / 2012 \\
17: 36: 37\end{array}$ & $\begin{array}{l}\text { 13/06/2012 } \\
07: 35: 34\end{array}$ & AF EP 4+ MAXI+ X44 Olympic 2012 & Baby Care & YES * & Delisting \\
\hline $\begin{array}{l}\text { 13/06/2012 } \\
11: 19: 14\end{array}$ & $\begin{array}{l}13 / 06 / 2012 \\
18: 42: 46\end{array}$ & AF EP 4+ MAXI+ X44 Olympic 2012 & Baby Care & NO : & Bad Reordering : \\
\hline $\begin{array}{l}13 / 06 / 2012 \\
18: 45: 03\end{array}$ & $\begin{array}{l}17 / 06 / 2012 \\
13: 38: 04\end{array}$ & AF EP 4+ MAXI+ X44 Olympic 2012 & Baby Care & NO : & Undefined \\
\hline
\end{tabular}

Data are stored in the system server and analysed to provide tailor made reports as in Figure 13 below.

Figure 13. Pie graphs discriminating motivations and promotions.

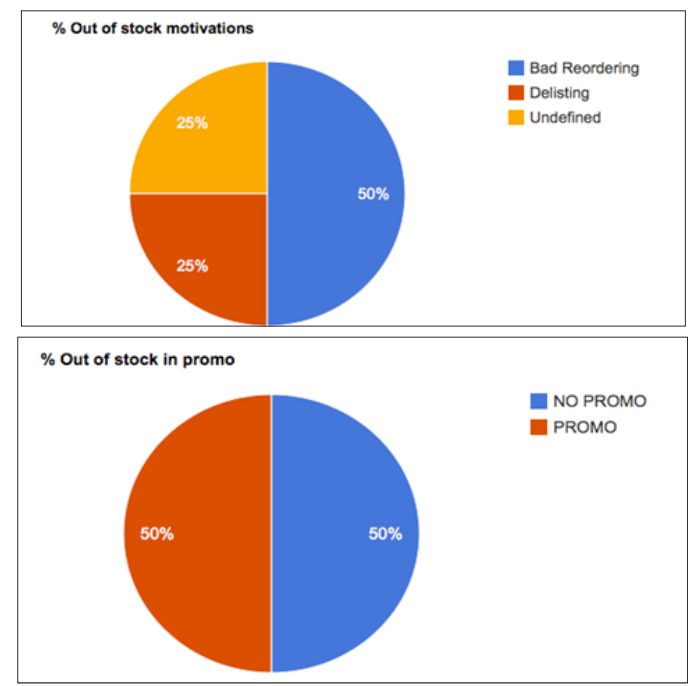

The two pie graphs attempt to indicate the SOOS motivations discriminating the SOOS related to promotional events from those due to other reasons. This functionality is really useful to have a clear view on the reason why the SOOS happened and can definitely support retailers and brands to work in the right direction to avoid SOOS.

\subsection{Comparisons between Time Periods}

The system is very useful as a diagnostic tool and it makes easier to decide the best strategy to make products available for shoppers. One of the most relevant aspects of the system is the opportunity to compare different periods, as well as measure the success of the in-store strategies deployed (Figures 14 and 15). 
Figure 14. Comparison between time periods considering two categories of product.

$\begin{array}{llll}\text { PREVIOUS PERIOD } & \text { FROM 07/08/2012 TO 07/31/2012 } & \text { PERIOD } & \text { FROM 06/03/2012 TO 06/23/2012 } \\ \text { PREVIOUS CATEGORY } & \text { Baby Care New WOT (9 SKU 27 FACING) } & \text { CATEGORY } & \text { Baby Care (8 SKU 18 FACING) } \\ \text { SOOS \% } & \mathbf{0 , 7} \% \text { (total hours O0S 21) } & \text { SOOS } \% & \mathbf{1 8 , 6} \% \text { (total hours 005 438) } \\ \text { AVG SOOS LENGHT } & \mathbf{1 0} \text { hours } 16 \text { min } & \text { AVG SOOS LENGHT } & \mathbf{6 2} \text { hours } \mathbf{3 7} \text { min } \\ \text { FULL STOCK INDEX } & \mathbf{9 5 , 4} \% & \text { FULL STOCK INDEX } & \mathbf{1 9} \%\end{array}$

Figure 15. Weekly comparison in rate of SOOS and estimate of SOOS.

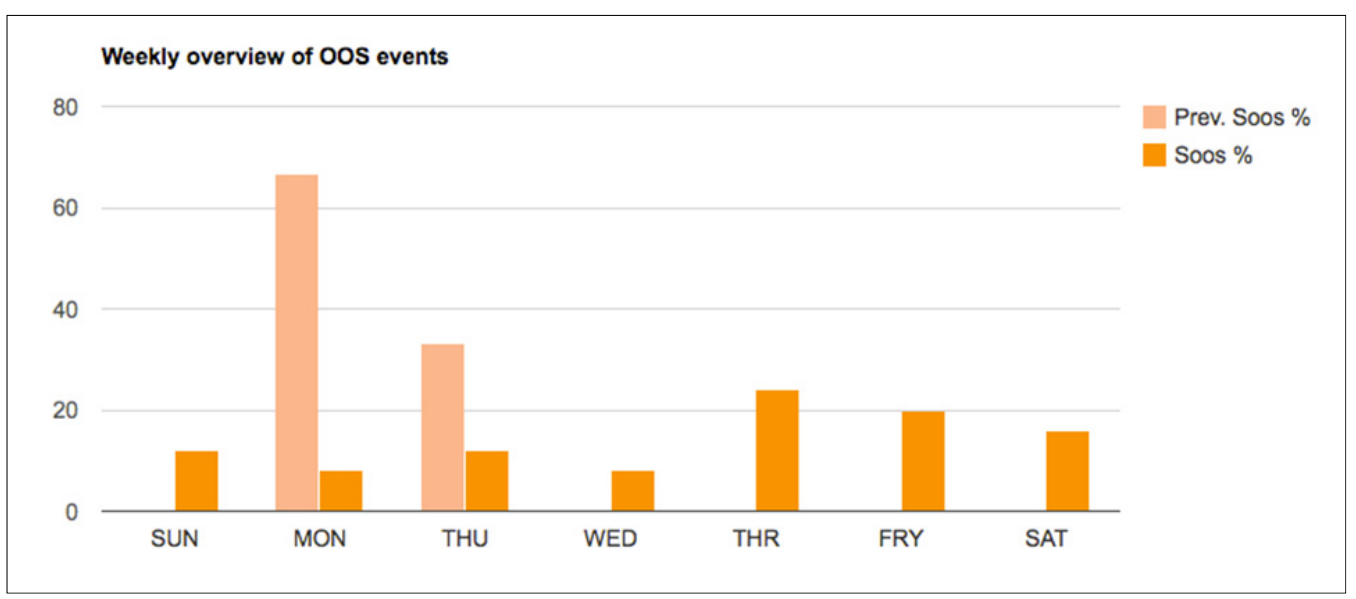

\section{Results}

The purpose of this paper is to propose an innovative idea of a system able to identify, in real time, OOS events. The Shelf Detector system intends to overcome limitations of previous methods introducing a real-time measure of OOS events that were installed in real retail environments to prove the overall feasibility and scalability. Proposed metrics also provided a good indication of the day by day use by store people. In this section, we describe two significant tests that are representative of the 50 installations of the systems all over the world.

The experimental results were obtained considering that each SKU is provided only in one place of the store and each product maintains its position in accordance to an "approved" planogram. This situation is not far from reality because planogram maintenance is one of the main topics of discussion between retailers and brands. Also, brands pay money to maintain a certain planogram position. For these reasons, the assumption of a quite stable planogram can be considered a real assumption for tests.

The Shelf Detector system was tested in our laboratories and then installed in several real stores, as mentioned above, and it showed a great versatility of installation on the shelf from the mechanical point of view. Also, data connections and data transmission were stressed in 18 months of real time data acquisition and transmission. Finally, we were also able to test the battery life in a real retail environment finding a battery lifetime of 12 months, for an average installation of 200 sensors connected with a battery powered RF (see Section 3.1 for details). This result is acceptable also from the scalability point of view and requires of retailers a very low maintenance effort.

Here, we report two significant tests that were performed in two different real stores to prove respectively the feasibility of the proposed system and its scalability. 
The first test was performed on 64 facing and 27 SKU using 64 sensors, a single RF transmitter and a Control Gear. During this test, data grabbed from Shelf Detector was compared with ground truth manually inspected in store and backed up with a photo of every out of facing and out of stock measured. Results of this first test demonstrated a high feasibility of the system with no false positive and a complete coherence with the ground truth. An example of a photo of this test where it is possible also to see sensors installed behind products is reported in Figure 16.

Figure 16. An example of OOS and a real image of sensors installed on the diapers shelf.

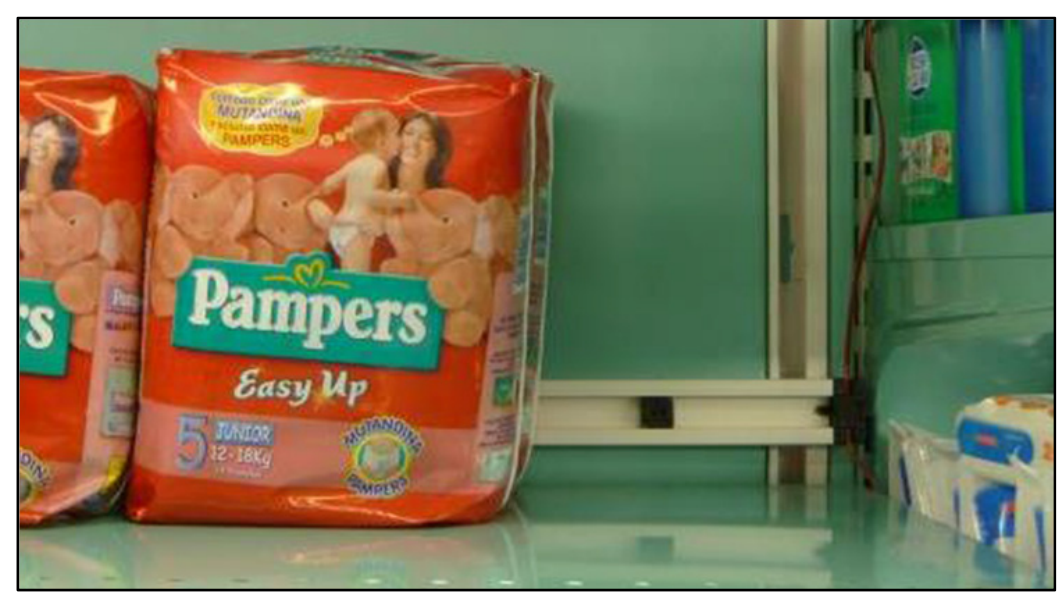

The second test was done to have a clear view on the scalability of the system and for that reason 678 sensors were used to measure the Shelf Detector System for 18 months. Five RF transmitters where used to collect data from sensors installed in five different categories and shelves inside the store. The control gear was responsible for collecting raw data and uploading changes of product status together with a time stamp to the cloud-based information collection and presentation system.

The systems behaved well and batteries lasted for the entire period. There were no significant problems in the second test and about 2500 out of facing and refill events were collected in the system. More than 3400 SOOS hours were analysed and reported in real time. The big amount of data allowed a complete scalability test both from the hardware and software point of view with interesting results from long time analysis. Some example of synthetic data and reports of this second test are shown in Figure 17. 
Figure 17. An example of OOS report with respect to average product availability (on shelf) and single SKU availability during the scalability test.

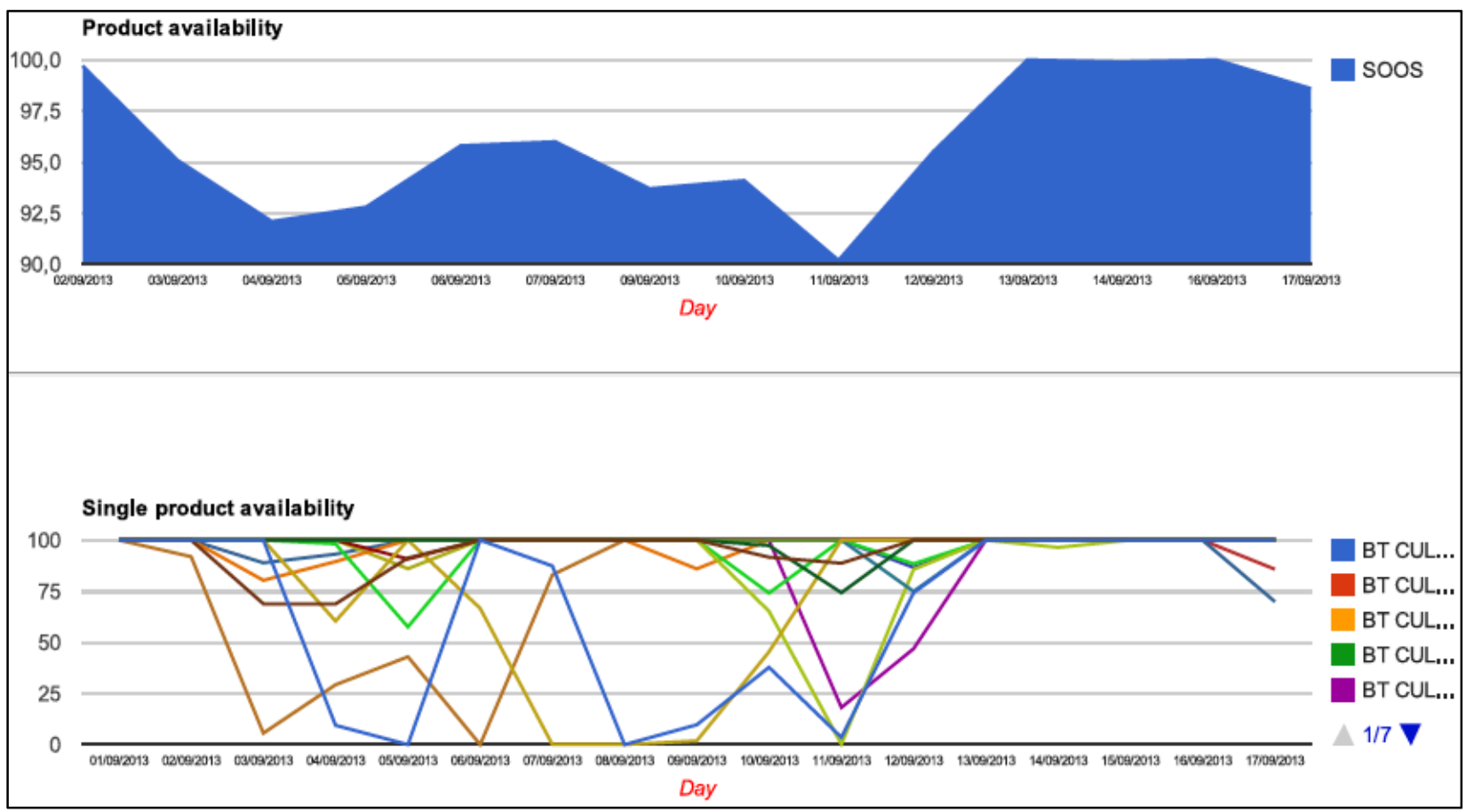

\section{Conclusions}

The purpose of this paper is to propose the innovative idea of a system able to identify, in real time, OOS events. The system named Shelf Detector intends to overcome limitations of previous methods introducing a continuous and real-time measure of OOS that has not been conceptualised until now. This study explores OOS values using time-variable definition, considering OOS events by day of the week, time of day and number facings. The metrics extracted by shelf detector system demonstrate how the system can introduce time-variable in the identification of unavailability of products on the shelf. So, we have introduced new metrics and displayed graphical representations of data extracted from the system. The system is also interesting for its industrial benefits and for the ability to send signals (SMS or similar) to the store team trying to interact with users to solve more effectively the SOOS problem and make products available for shoppers. The field of SOOS data is making much progress, with the insights here presented, and further results coupling geographical data (stores in different areas) and different categories can also increase knowledge on the problem. In this research, our aim has been to introduce new metrics that take into account the time variable, and to report more significant results that are testament to the ability of the system.

Moreover, results from extensive tests will be presented to demonstrate the possible applications of the system in the field of information mining for SOOS and decision support systems applied to real stores. Future work in this field will be focussed on data classifications to better understand abnormal behaviours, learning from data series in order to have a powerful decision support system for shelf operations. 


\section{Acknowledgments}

The authors thank all the technical staff from Grottini Lab for the precious support and for all discussions and suggestions on this special topic; thanks also go to all the people we have met during operations who have helped us to improve the system and have shared knowledge about stores and shelves.

\section{Author Contributions}

Emanuele Frontoni and Valerio Placidi are responsible for the concept of the paper, a large part of the results presented and SOOS analytics aspects. Adriano Mancini and Primo Zingaretti contributed to the mathematical soundness of the theory, data analytics aspects and the writing. All authors have read and approved the final published manuscript.

\section{Conflicts of Interest}

The authors declare no conflict of interest.

\section{References}

1. Zinn, W.; Liu, P.C. Consumer response to retail stockouts. J. Bus. Logist. 2001, 22, 49-71

2. Roland Berger Strategy Consultants. ECR-Optimal Shelf Availability: Increasing Shopper Satisfaction at the Moment of Truth. Available online: www.ecr-all.org/files/ pub_2003_osa_blue_book.pdf(accessed on 21 April 2014).

3. Aastrup, J.; Kotzab, H. Analyzing out-of-stock in independent grocery stores: An empirical study. Int. J. Retail Distrib. Manag. 2009, 37, 765-789.

4. Aastrup, J.; Kotzab, H., Forty years of out-of-stock research—and shelves are still empty. Int. Rev. Retail Distrib. Consum. Res. 2010, 20, 147-164.

5. Gruen, T.W.; Corsten, D.S.; Bharadwaj, S. Retail Out-Of-Stock: A Worldwide Examination of Extent Causes and Consumer Responses. Available online: www.knowledge.emory.edu/ papers/1030.pdf (accessed on 21 April 2014).

6. Campo, K.; Gijsbrechts, E.; Pisol, P. Towards understanding consumer response to stock-outs. J. Retail. 2000, 76, 219-242.

7. McKinnon, A.C.; Mendes, D.; Nababteh, M. In-store logistics: An analysis of on-shelf availability and stockout responses for three product groups. Int. J. Logist. Res. Appl. 2007, 10, 251-268.

8. Coca-Cola Retailing Research Council, Andersen Consulting. Where to Look for Incremental Sales Gains: The Retail Problem of Out-of-Stock Merchandise. Available online: http://ccrrc.org/wp-content/uploads/sites/24/2014/02/Where_to_Look_for_Incremental_Sales_Gains_ The_Retail_Problem_of_Out-of-Stock_Merchandise_1996.pdf (accessed on 21 April 2014).

9. Chandon, P.; Hutchinson, J.W.; Bradlow, E.T.; Young, S.H. Does in-store marketing work? Effects of the number and position of shelf facings on brand attention and evaluation at the point of purchase. J. Mark. 2009, 73, 1-17. 
10. Nenycz-Thiel, M.; Beal, V.; Ludwichowska, G.; Romaniuk, J. Investigating the Accuracy of Self-Reports of Brand Usage Behavior. J. Bus. Res. 2012, 66, 224-232.

11. Hausruckinger, G. Approaches to Measuring On-Shelf Availability at the Point of Sale; ECR Europe White Paper; Available online: http://ecr-all.org/files/OSA-Measurement-White-Paperfinal_E1.pdf (accessed on 21 April 2014).

12. Frontoni, E.; Raspa, P.; Mancini, A.; Zingaretti, P.; Placidi, V. Customers' activity recognition in intelligent retail environments; Lecture Notes in Computer Science; Springer: Berlin/Heidelberg, Germany, 2013; Volume 8158, pp. 509-516.

13. Frontoni, E.; Mancini, A.; Zingaretti, P.; Placidi, V. Smart vision system for shelf analysis in intelligent retail environments. In Proceedings of 2013 ASME/IEEE International Conference on Mechatronic and Embedded Systems and Applications, Portland, OR, USA, 4-7 August 2013; doi:10.1115/DETC2013-12317.

14. Frontoni, E.; Mancini, A.; Zingaretti, P. RGBD Sensors for human activity detection in AAL environments. In Ambient Assisted Living: Italian Forum 2013; Longhi, S., Siciliano, P., Germani, M., Monteriù, A., Eds.; Springer: Berlin/Heidelberg, Germany, 2014.

15. Catani, L.; Frontoni, E.; Raspa, P.; Zingaretti, P. A framework based on vision sensors for the automatic management of exchange parking areas. In Proceedings of 2010 IEEE/ASME International Conference on Mechatronic and Embedded Systems and Applications (MESA 2010), Qingdao, Shandong, China, 15-17 July 2010; pp. 319-324.

16. Catani, L.; Frontoni, E.; Raspa, P.; Zingaretti, P.; Di Pasquale, G. Efficient traffic simulation using busses as active sensor network. In Proceedings of the ASME Design Engineering Technical Conference, Washington, DC, USA, 28-31 August 2011; pp. 889-894.

(C) 2014 by the authors; licensee MDPI, Basel, Switzerland. This article is an open access article distributed under the terms and conditions of the Creative Commons Attribution license (http://creativecommons.org/licenses/by/3.0/). 